\title{
LA ILUSIÓN ANAMNÉTICA EN LAS FICCIONES NARRATIVAS RECIENTES: SOBRE MEMORIAS, TRAUMAS Y TESTIGOS
}

\author{
THE ANAMNETIC ILLUSION IN THE CURRENT NARRATIVE \\ FICTIONS: ABOUT MEMORYS, TRAUMAS AND WITNESSES
}

\author{
Sandra Beatriz Navarrete Barría \\ Pontificia Universidad Católica de Valparaíso. Valparaíso, Chile \\ sandra.navarrete.b@gmail.com
}

Recibido: 22.05.2013. Aceptado: 16.09.2013.

Resumen: El presente artículo aborda el problema de la memoria en las ficciones narrativas del trauma reciente, en Argentina y Chile, preguntándose por las relaciones y diálogos que establece este género con los de tipo referencial, si pensamos en el rol fundacional que cumplen la autobiografía y el testimonio respecto del ejercicio narrativo y estético de la memoria. En base a lo anterior, nuestra hipótesis postula que las ficciones de la memoria tienen como factor común lo que denominaremos 'ilusión anamnética', es decir, aquellos procesos discursivos de evidenciación del trabajo memorialístico, producidos en el texto literario de ficción. De este modo, la ilusión anamnética se erige en el cruce entre ficción, referencialidad y memoria traumática, construyendo un inestable espacio de autonomía.

Palabras clave: Memoria, trauma, ficción narrativa, géneros referenciales.

\begin{abstract}
This article addresses the problem of memory in recent narrative fiction dealing with trauma in Argentina and Chile, questioning the relations and dialogues that this genre establishes with those of referential type, considering the foundational role that autobiographies and testimonies play in the narrative and aesthetic exercise of memory. Our hypothesis postulates that fictions dealing with memory have as a common factor what we will designate 'anamnetic illusion', that is to say those discursive processes of evidenciation of memoralistic work produced in fictional literary work. In this way the anamnetic illusion develops at the intersection of fiction, referenciality and traumatic memory, a space of unstable autonomy.
\end{abstract}

Key words: Memory, trauma, narrative fiction, reference genres. 
El designio: escuchar, en el silencio que impone la escritura, esas voces que empezaron a hablar cuando leí el diario de Sonia, buscar las huellas personales en la masa de acontecimientos, trazar junto con esa mano que se aferra a las palabras para resistir, trazar con Sonia. ¿Estamos ya en la literatura? ¿La novela va a ser la forma de representar una historia que se entreabre cada vez que se la roza?

(Tununa Mercado, 2003: 144)

\section{Ficciones narrativas de la memoria y el trauma}

L L Amplo corpus de novelas que recogen la experiencia traumática de de los noventa hasta hoy, nos obliga a observar este gesto escritural como novedoso e interesante, en la medida que proponen una nueva relación narrativa de la memoria social con la literatura, a través de distintos modos discursivos de trabajar la verdad y la ficción.

La preocupación por las novelas argentinas que trabajan el código memorialístico ha sido una de las ramas más destacadas de la crítica reciente, observándose un común acuerdo en cuanto al devenir de este tipo narrativo. En un primer momento, durante la dictadura misma, surgen ficciones narrativas que abordan la temática de la represión, la tortura y los desaparecidos, no a partir de la denuncia, sino que con el motivo de promover una reflexión crítica sobre los eventos contingentes del país, a cargo de un lenguaje opaco, artificioso y ambiguo. Como explica Gramuglio (2002), estas obras "transformaban o directamente eludían las convenciones de la mimesis tradicional" proponiendo "verdaderos ejercicios de desciframiento, de lectura entre líneas, para unas historias y unos personajes dotados a veces de fuerte carga simbólica o alegórica” (10). A este período pertenecen autores como Osvaldo Soriano, Juan José Saer y Ricardo Piglia. Posteriormente, los estudios definen una etapa en donde prima el relato testimonial/documental, los que tienen como principal referente el Informe del 'Nunca Más' de la CONADEP y el juicio a las Juntas Militares en 1985. Esta literatura, escrita en su mayoría por sobrevivientes y ex militantes, se caracterizó por el objetivo de denuncia. Aquí encontramos Preso sin nombre, celda sin número (1982), de Jacobo Timerman o The little school (1986) de Alicia Partnoy. Esta modalidad se extiende a los noventa, modulándose la función testimonial con la poética en obras híbridas, como por ejemplo, la 
novela testimonial de Nora Strejilevich (1997) Una sola muerte numerosa o Un hilo rojo (1998) de Sara Rosenberg.

Paralelamente, comienzan a publicarse novelas como El fin de la historia (1996) de Liliana Heker, Mala junta (1999) de Paoletti y Los planetas (1999) de Sergio Chejfec, obras que contrastan profundamente con los corpus iniciales. Estas nuevas ficciones de la memoria proponen "narrar refiriendo por completo y de modo directo, los sucesos y acciones más atroces o inenarrables" (Dalmaroni, 2004: 159). Por otro lado, se contraponen también a las producciones de la etapa documental/testimonial de los 80, en la medida que "no persiguen la verdad objetiva del documento, ni la verdad subjetiva del testigo o participante que ha sobrevivido a los hechos", sino que buscan "un horizonte de comprensión más ancho y penetrante que el del dato y el de la información" (Di Marco, 2003: 5).

En el caso de Chile, los estudios literarios convocan los siguientes temas: la narración del trauma (Lazzara, 2007); los problemas del duelo y la derrota (Avelar, 2000); el testigo y el testimonio (Peris Blane, 2009); la memoria, la diáspora y la genealogía familiar (Cánovas y Sherman, 2008); la inscripción de la memoria de dictadura en la actualidad (Carreño, 2009), entre otros. En el campo específico de la narrativa ficcional, la crítica destaca nombres como José Donoso o Diamela Eltit, autores claves reflexionan sobre los cambios impuestos por la dictadura, en la dictadura misma. La novela Casa de campo (1978) de José Donoso, por ejemplo, es catalogada como "el intento definitivo por un novelista, no tradicionalmente considerado muy político, de elaborar el trastorno sufrido por Chile durante los setenta" (Avelar, 2000). A su vez, Lumpérica de Eltit, clasificada por algunos como la anti-novela de la dictadura, se erige como la propuesta más original y compleja de la escena contemporánea. Esta obra representa de modo ejemplar la estética de la fragmentariedad (Lazzara, 2007) y de lo residual (Richard, 1998). Otras tipologías son aquellas que Strejilevich (2006) define como lejanas del trauma, intimistas, pero aún relacionadas con el golpe. Algunos títulos son Coral de guerra (1979) de Fernando Alegría, La casa de los espíritus (1982) de Isabel Allende, En este lugar sagrado (1977) de Poli Délano, entre otras ${ }^{1}$.

${ }^{1} \mathrm{Al}$ mismo tiempo que se producía una transformación sustantiva en las ficciones narrativas, el testimonio cobraba una importancia inusitada. Aparecen títulos como 
En resumen, en cada país distinguimos primeramente un corpus de ficciones 'alegóricas' ${ }^{2}$ (Avelar, 2000) que aluden oblicuamente a un referente cuestionado. En un segundo período, apreciamos una estética de la exposición, en donde la memoria se arraiga en la voz testimonial y se completa en los soportes documentales, para levantar la figura del testigo y asumir una perspectiva inquisidora frente a las políticas del olvido. Finalmente, en una tercera etapa, nos enfrentamos con textos que abordan el problema de la memoria inmediata, variando entre tres elementos: la documentalidad del testimonio, la narración personal del sujeto que rememora y la crítica a la autonomía estetizante de la novela alegórica. De esta manera, se posicionan desde una focalización más realista, pero que levanta la ficción como un lugar válido para el ejercicio de la rememoración. Estamos ante un conjunto de 'ficciones que hacen memoria', las que tienen la particularidad de "asumirse como ficciones literarias que, como tales, privilegian el papel de la invención de tramas ficticias y el trabajo estético sobre el propio lenguaje" (Di Marco, 2003: 3).

Nuestro artículo parte de la base de que estas novelas aportan a la construcción de la memoria colectiva, posicionadas desde el código narrativo ficcional, apropiándose de un espacio escritural que parecía conquistado por las 'escrituras del yo' (Miraux, 2005), abarcando, de esta manera, dos niveles de intervención discursiva: el nivel social de la memoria traumática, al recrear eventos históricos de alcance colectivo, y el nivel propiamente literario, situándose frente al canon memorialístico y reactualizando el género de la ficción.

Ahora bien, y en atención a lo dicho por la crítica, cabe preguntarse si

Tejas verdes (1974, España; 1995, Chile) de Hernán Valdés, catalogado como el primer testimonio de denuncia en el exilio. A pesar de que el mismo autor insiste en su calidad de testimonio, la obra se fundamenta en la ficción de un diario escrito en cautiverio (Strejilevich, 2006). Otras obras coetáneas de este género son Cerco de púas (1976), de Aníbal Quijada, Un día de octubre en Santiago (1980) de Carmen Castillo y Mis tres primeros minutos (1989) de Emilio Rojas. La 'novela documental' también es una variable presente en esta época, específicamente desde el exilio, donde podemos destacar títulos como El paso de los gansos (1975) de Fernando Alegría, Actas de Marusia (1977) de Patricio Manns, Soñé que la nieve ardía (1975) de Antonio Skármeta y Abel Rodríguez y sus hermanos (1981) de Ana Vásquez.

${ }^{2}$ Avelar (2000) explica que la alegorización tiene lugar cuando aquello que es más familiar se revela como otro (316). 
la condición de 'ficciones de la memoria' radica únicamente en la representación del conflicto de la memoria traumática. En otras palabras, ¿el núcleo semiótico de la novela, anclado en el pasado referido, es el pilar que le otorga el carácter memorialístico al texto literario? Creemos que el hecho traumático recreado no es el único soporte para la memoria, ya que si bien, el espectro extratextual configurado en estas obras apela a una memoria social evidente, estas novelas se sostienen en un ejercicio formal específico, en el que se destacan procesos discursivos nomádicos que van evidenciando, en la progresión narrativa, un ejercicio memorialístico particular, el que a continuación acotaremos teórica y analíticamente.

\section{Las voces del trauma: Hacia una tipología del testigo}

Para Di Marco (2003), las 'ficciones que hacen memoria' son caracterizadas por la politización de la memoria, a través de la politización de la escritura. En este proceso, el rol de la verdad recuperada es el cuestionamiento por su alcance en nuestro presente.

La ficcionalización de este pasado casi presente (un pasado no del todo pasado, "un pasado que no quiere pasar") debe tomarse menos como un ensayo de exactitud y fidelidad, una pura retrospección historicista, que como una "terapéutica", un trabajo de la memoria tendiente a (re) escribir de forma creadora las heridas y los trastornos ocasionados por la historia y sus terrores (Di Marco, 2003: 10).

La escritura memorialística como elaboración de un trauma colectivo reciente, una escritura que se hace cargo del evento traumático desde la arista creativa y ficcional, que vuelve ambiguo el archivo memorial representado. Ahora bien, si a la fragmentariedad de la memoria le agregamos el elemento afectivo del 'trauma's, esta irrepresentabilidad se expande aún

${ }^{3}$ Trauma es definido por La Capra (2005) como una experiencia específica y perturbadora, que es capaz de arrasar con la experiencia de tipo cotidiana, que le da un sentido de integridad y articulación a la vida del individuo. La destrucción de la estructura, más o menos estable de la vida, se produce al disociarse la cognición del afecto en el individuo y su relación con el entorno sociocultural, esto es, se desesta- 
más y, por lo tanto, aumentan a su vez los huecos que actúan como una suerte de poros por donde se permean los elementos ficcionales. El trauma, según La Capra (2005), está definido por la constante tensión entre síntomas como el acting-out ${ }^{4}$-en donde el sujeto del trauma no es capaz de evocar el acontecimiento límite, sino que lo revive- y la necesidad de elaboración del mismo trauma, entendida como aquel esfuerzo por volver a articular los afectos y las representaciones personales, lo que al mismo tiempo, en un nivel no-personal, permite la acción ética y sociopolítica de una colectividad en el presente y futuro ${ }^{5}$. La interrogante que aflora es la siguiente: ¿cómo es posible una 'elaboración' narrativa del trauma que implique a su vez la textualización de un acting-out, supuestamente inefable? Nuestra hipótesis al respecto se sustenta en el cruce entre la existencia de un 'testigo' y un 'autor'. En otras palabras, la posibilidad de la presencia del acting-out en la escritura, que la convierte en una escritura del trauma, se genera sólo si es otro sujeto distinto del que ha experimentado el acontecimiento límite quien la construya. Este sujeto-otro se apropia de la experiencia traumática, ya sea mediante relatos o representaciones simbólicas de la misma, o al compartir directamente con el sujeto del trauma. En esta apropiación virtual del evento traumático, se debe aprehender el actingout, de modo tal de poder representarlo.

En base a lo anterior, proponemos tres tipos de testigos que nos per-

bilizan las expectativas y la comprensión de los contextos, generándose una fractura irreconciliable. En definitiva, quien padece de un trauma, "siente, desconcertado, lo que no puede representar o representa anestesiado lo que no puede sentir" (La Capra, 2005: 64).

${ }_{4}^{4}$ El acting-out es un síntoma postraumático que se puede entender como el opuesto a la 'elaboración'. "En el 'acting-out', los tiempos hacen implosión, como si uno estuviera de nuevo en el pasado viviendo otra vez la escena traumática. Cualquier dualidad (o doble inscripción) del tiempo (pasado y presente, o futuro) se derrumba en la experiencia" (La Capra, 2005: 46), por ello podemos decir que, a través de la elaboración del trauma, el individuo puede distinguir entre pasado, presente o futuro.

${ }^{5}$ Para el autor, la elaboración del trauma es fundamental, no sólo a un nivel individual, sino que también sociocultural, pues existe claramente una dimensión colectiva de los traumas históricos que, en ciertas etapas de la construcción memorialística, los convierte en eventos sacralizados, absolutos, fundantes o sublimes. Esta fidelidad ética al trauma la desarrollan frecuentemente los testigos que se niegan a olvidar a las víctimas a través de la resistencia a 'elaborar' su trauma en cualquier tipo de representación. De esta manera, la ‘elaboración' es observada como una suerte de traición al trauma, ante lo que habría que resistirse, según plantea La Capra (2006). 
mitirán ubicar en coordenadas concretas esta categoría y así alejarnos de definiciones hiperbólicas. En primer lugar, consideramos al testigo que es el 'sujeto del trauma' propiamente dicho: el 'testigo-sobreviviente', aquel teorizado por Agamben (2000) ${ }^{6}$. En segundo lugar, advertimos un tipo de testigo que no ha vivido la experiencia del trauma, pero que, sin embargo, accede a ella al conocer directamente al sobreviviente. Aquí podemos encontrar a los familiares, pero también a quienes entrevistan a los sobrevivientes, $y$, en general, a investigadores de diversa índole que logran acceder a la primera fuente testimonial: el 'testigo-contactual'. Y en tercer lugar, podemos definir aquel testigo que se relaciona con las representaciones simbólicas del evento traumático, ya sea a través de narraciones históricas, literarias, testimoniales, visuales, entre otras, estableciendo un vínculo específico con ellas: 'testigo-simbólico'. Cada una de estas tipologías son también tres niveles factibles de narración testimonial, que van de mayor a menor intensidad de apropiación de la experiencia traumática, apropiación que es inversamente proporcional a la posibilidad de escritura del trauma. Es decir, a mayor cercanía con el trauma, menor facilidad de expresión, representación o transmisión del mismo hacia otro.

Ahora bien, ¿cómo podemos observar las relaciones entre el 'testigoautor', el trauma y la ficción en una novela de la memoria concreta? Estimamos que de dos modos narrativos básicos: en primer lugar, el 'testigoautor' como figuración propiamente tal, ya sea a partir del narrador como reflejo sugerente de un testigo extraliterario, que muchas veces coincide con el autor, o bien como un personaje inserto en el mundo ficcional, que se define a partir de su condición de 'sujeto del trauma', y que se encuentra posicionado en la encrucijada entre la elaboración y la permanencia del daño traumático. Y en segundo lugar, apreciamos también un trabajo del tiempo y el espacio como ejes impregnados de lo testimonial, en donde las descripciones de lugar y los modos de trabajo de lo temporal convierten

${ }^{6}$ Agamben entiende al testigo sobreviviente como el puente entre el Muselmann y la comunicación del testimonio hacia otros sujetos. El Muselmann es un tipo de víctima que se transforma en el verdadero testigo del evento traumático, justamente, gracias a ser totalmente destruido, es decir, la víctima que muere en el evento traumático es el testigo real. Por esta razón, no puede dar testimonio. Se necesita entonces, un 'testigo-otro', un sobreviviente. 
estos ejes en una suerte de testigos materiales de la memoria, que si bien no elaboraban escriturariamente el acontecimiento límite, igualmente irradian huellas de un trauma fragmentado.

\section{El papel de los géneros referenciales}

Cuando pensamos en la ficción y su relación con las 'escrituras del yo', estamos acotando un problema de apropiación discursiva por parte de la novela, el que se debe considerar enmarcado dentro de un fenómeno de retroalimentación estética de mayor envergadura, aquel que va permitiendo las transformaciones evolutivas de los géneros literarios. De este modo, se configura un espacio de interferencia entre las ficciones, autobiografías y testimonios, espacio que apela a una memoria colectiva y sus múltiples voces enunciativas, pero también a un canon literario que, como tal, está sustentado en "criterios inestables y cambiantes a través del tiempo, por más que puedan parecer eternos en un momento histórico dado" (Pratt, 2000: 71).

En este sentido, nos resulta pertinente la concepción de género literario como marcos discursivos (Mignolo, 1986), por los cuales entenderemos una serie de conocimientos empleados para producir e interpretar discursos. Estos marcos son el producto de sistemas de codificación que posibilitan la organización de la información de tipos o clases de discursos7.

En base a lo anterior, las ficciones narrativas de la memoria se observan como un grupo de textos literarios que se apropian de algunos mecanismos o procedimientos que figuran dentro de un marco discursivo diferente al de la novela y que logran situarse con fuerza en el boom de producción

${ }^{7}$ Nos inclinamos por esta noción, ya que, al no imponer límites textuales a los distintos tipos de discursividad literaria, se convierte en una categoría flexible que nos permite abordar estas novelas, justamente en el cruce de diferentes modos discursivos. Mignolo (1986) establece 'marcadores genéricos' que se manifiestan como dominantes y que pueden agrupar ciertos textos, pero nunca de manera estricta. Esto significa que un texto literario puede desarrollar más de un marco discursivo y cruzar los códigos respectivos. Estos hibridismos textuales se producen, según Mignolo, a causa de los frutos de la invención humana. Nosotros nos permitimos agregar, que importan también la voluntad de transgresión de los esquemas, además de la reinvención de los mismos, influenciados por los distintos elementos del sistema literario (contextos de producción, expectativas de recepción, intereses editoriales, etc.). 
memorialística. En este sentido, cabe preguntarse por qué estos géneros se asocian más fácilmente al trabajo de rememoración que otros. La autobiografía y el testimonio, como relatos de vida construidos por el mismo sujeto de la experiencia, parecen óptimos para desarrollar un ejercicio de recordación, al posicionarse el narrador/autor/personaje, desde un presente de enunciación y, desde allí, volcarse hacia escenas del pasado, tejiendo un hilo conductor que las discrimina.

Por otro lado, tenemos el factor de realidad de estos géneros, ya que apelan a una suerte de recuperación de la verdad, reforzada por la mismidad del autor y el narrador/personaje. Dicha verdad recuperada se obtiene mediante el trabajo de la memoria narrativa desplegada en el texto mismo, que genera una especie de espejismo referencial, en el cual los elementos extratextuales se reflejan en los propios del texto. Si bien ambas formas narrativas apelan a la verdad recuperada, ciertamente poseen connotaciones diferentes. En el caso de la autobiografía, se construye una verdad subjetiva respaldada en la firma del autor, por lo tanto, las estrategias narrativas deben reforzar la imagen del autor/narrador/personaje como honesto, sincero, auténtico, etc. (Miraux, 2005). En el caso del testimonio, la verdad trabajada en la narración involucra la sensibilidad de un pueblo, contribuyendo, de este modo, a la articulación escrita de la memoria colectiva, a través de uno de sus protagonistas (Ochando, 1998). Por lo demás, no es tanto la imagen del autor lo que se evalúa como su relato, el cual siempre será objeto de ‘juicio’. Por ello, generalmente, los testimonios se respaldan con los denominados 'soportes textuales': documentos, textos periodísticos, fotos, etc., que van reforzando la verdad de lo testificado. La literatura testimonial, en el gesto discursivo de recuperar la verdad, introduce en el campo de los textos literarios elementos que habían sido menospreciados por la literatura culta moderna, como lo son la oralidad, el relato no pulido de la vivencia personal y/o de la urgencia social y, en definitiva, un hecho que es factible de documentar (Volek, 2000) ${ }^{8}$.

Finalmente, cabe mencionar que el testimonio, al ser expresión del

${ }^{8}$ Para Volek (2000), el testimonio tenía como aspiración llegar a ser la 'historia viva', una suerte de documento hablado en primera persona, por lo cual la historia colectiva no es tanto un trasfondo del relato como su objeto mismo, siendo justamente esta la característica que lo separaba radicalmente de otras escrituras del 'yo', como la autobiografía o las memorias. 
trauma, siempre será problemático debido al factor afectivo que encierra, más aún si es un trauma colectivo, poniendo en entredicho la supuesta referencialidad del testimonio. Como explica Felman (1992), en el trauma masivo todos los marcos de referencialidad colapsan ante "hechos que no se organizan en una comprensión o en un recuerdo, actos que no se pueden (...) asimilar en un saber acabado" (citado en Strejilevich, 2006: 17). En este sentido, podemos apreciar que, en las ficciones narrativas de la memoria reciente, lo que nos acerca a la categoría de memoria no es lo rememorado, o sea, escenas del pasado, sino que más bien, el cómo se ha llegado al objeto rememorado, en otras palabras, cómo se produce el fenómeno estético de la 'rememoración', característica que resulta justamente de la apropiación de distintos niveles de las escrituras del yo, ancladas en la figura del testigo.

\section{Apuntes para un posible corpus}

Como postula Martínez Bonati (2001), las ficciones narrativas se oponen a los géneros referenciales al no funcionar a partir de 'pactos de veracidad' que vuelquen al lector sobre la coincidencia de los espacios, personajes o acontecimientos con la realidad extratextual. Sin embargo, en las ficciones de la memoria observamos que, si bien no apelan abiertamente a la 'veracidad' de lo narrado, claramente desarrollan una tensión entre realidad y ficción, situándose justamente en el conflictivo territorio que las limita, construyendo pilares hacia la autonomía ficcional, pero también habilitando puentes hacia lo extradiegético. Es este espacio fronterizo el cual les permite establecer distintos mecanismos de lo ambiguo, bien apropiándose directamente de ciertas propiedades de la autobiografía o el testimonio, en el gesto de la validación, o bien desvirtuándolas y juzgándolas.

Es así como en las novelas argentinas de Liliana Heker y María Teresa Andruetto nos encontramos con una serie de desplazamientos de las categorías de ‘testigo', 'víctima’ y ‘traición' a partir de una ilusión anamnética cuestionada. En el caso de El fin de la historia (2004) de Heker advertimos una apuesta estética que utiliza la ficción como un elemento que desestabiliza las construcciones idealizantes sobre la relación víctima/victimario, situando al primer concepto en un umbral heroico resquebrajado. En esta 
obra observamos tres niveles narrativos: en primer lugar, el relato que nos cuenta cómo Diana Glass intenta escribir su novela (narrador omnisciente); en segundo lugar, fragmentos de la novela que está escribiendo Diana Glass, indicados en letra cursiva (en primera persona, en la voz de la misma Diana-personaje), y finalmente el relato de la vida de Leonora Ordaz (narrador omnisciente). Tanto en el primero como en el segundo nivel observamos una referencialidad tanto colectiva como individual, ambos estratos dejan entrever el contexto sociopolítico de violencia y represión que definió a la Argentina del '76, pero además, la voz de Diana se transforma en alter ego de la autora Heker. De este modo, en la intención de retratar el sentir de una época histórica concreta, el texto hace suyo al testimonio, y de la misma manera, en el gesto de desdoblamiento de la autora en su yo-fictivo, Diana Glass, la novela se acerca a las propiedades de la autobiografía, y además al de la biografía, si pensamos en la amistad extratextual entre la autora y Lucy ${ }^{9}$. En suma, si bien se aprecia, por un lado, la reducción de la distancia entre autor/narrador/personaje, para apelar a la veracidad de los hechos relatados y, por otro, la subjetivización de los personajes y el narrador, el cual, al igual que en las escrituras del yo, construye una autoimagen confiable, dicha configuración se tergiversa al exacerbarse el idealismo de la autora. La intención de Diana Glass de elaborar una suerte de 'memoria emblemática' (Stern, 2000) se ve truncada cuando se le produce el revelamiento de la verdad de su compañera. Uno de los rasgos más importantes del testimonio es que el ‘testigo' juzgue como éticamente inaceptable lo testificado, y es en este momento de develación de la traición, cuando el juicio moral apenas se disimula. "De pronto estaba ahí con su sonrisa inalterable hasta la repugnancia, sentada ante mis ojos en una mesa de la Richmond y hablándome del proyecto popular de un Almirante y de sus arrullos de torcaza con un torturador" (Heker, 2004: 276). Dicho hito coincide con la muerte del personaje en cuestión, en la medida que, para el deseo escriturario de Diana, Leonora como 'delatora' ya no es digna de ser un personaje literario, al menos no el suyo.

9 "Lucy" es el nombre por el que se conoce a Mercedes Carazo, representada por el personaje de Leonora en la novela de Heker. Tal como se relata en la obra, Lucy fue una montonera que cae presa y se enamora de Antonio Pernías, uno de sus victimarios en la ESMA, y luego se convierte en colaboradora 'intelectual', como recalca la misma autora. 
La mujer en cuestión de María Teresa Andruetto también actualiza las categorías de 'testimonio' y 'víctima', pero desde una perspectiva relativista, eliminando juicios éticos, al estar a cargo de un narrador de función objetiva, sustentado en la discursividad, en base al formato del 'informe' de investigación. El 'informante'-narrador principal- es quien va armando el entramado discursivo gracias a la información recogida, los testimonios y los breves comentarios personales que se permite. Los comentarios pueden ir dentro de la narración principal, o bien, entre paréntesis, indicados con la abreviatura "N. del I." (Nota del informante), como en el siguiente ejemplo: "Se hace necesario recordar que se está hablando de los años setenta (N. del I.: considérese que entre 1964 y 1969, Eva cursa sus estudios secundarios y en enero de 1970, cuando tiene dieciocho años, se va de su pueblo a la ciudad)" (Andruetto, 2009: 51). Por otra parte, tenemos los testimonios, los que están citados fragmentariamente y no se extienden más allá de dos líneas (excepto el testimonio final de Eva). Los testimonios refuerzan la investigación y el cometido del 'informante' en términos de veracidad, pero también van armando un contexto para la 'mujer en cuestión', una red de relaciones interpersonales que el narrador divide en familiares, amistades y conocidos circunstanciales. De esta manera, nos acercamos ineludiblemente al panorama de época que rodea a estas personas, pero sólo de manera incompleta y fracturada, pues en ningún punto específico (maternidad, vida matrimonial, estadía en la cárcel, entre otros) se llega a un conocimiento acabado, fundamentalmente porque los mismos testimonios no concuerdan en plenitud, y aún más, muchas veces muestran versiones encontradas para un mismo hecho. Para reforzar la verosimilitud, se despliega un tercer nivel que incluye notas al pie de página y la alusión a archivos adjuntos (21 casetes, fotocopias de cartas y fotografías). La notas versan sobre aclaraciones de fechas, hechos históricos e individuales de los testigos: "Murió de un infarto de miocardio el 27/10/76, dos días después de haber sido detenida Eva” (Andruetto, 2009: 23). También son utilizadas para definir palabras o términos propios de Argentina o del período militar: "Las fuerzas policiales de la época utilizaban automóviles Ford, modelo Falcón" (Andruetto, 2009: 17). En definitiva, la verosimilitud se trunca en el momento en que el objetivo principal de la investigación se ve frustrado: conocer a la 'mujer en cuestión', en las distintas etapas de su vida, para comprender cómo llega a cometer 'aquello', la traición. En otras 
palabras, el aparataje de referencialidad resulta un exceso irónico para el conocimiento que se pretende obtener pues, finalmente, dicha verdad no se descubre por completo.

En las novelas chilenas, distinguimos una inclinación por la perspectiva infantil para desarmar las construcciones memorialísticas oficiales. Estas voces infantes trabajan la memoria desde un 'yo' inseguro, inacabado, que está en un proceso identitario marcado por la marginalidad de la memoria familiar. En el caso de Costamagna (1996), En voz baja, el relato de Amanda se basa en la indagación del paradero de su padre desaparecido, de este modo, la imposibilidad de acceder a la memoria de éste radica justamente en su ausencia. La niña no hereda el trauma del padre, sino que, a raíz del sentimiento de carencia paterna, va creciendo en ella un deseo de fusión con el padre, que en ocasiones adquiere ribetes edípico-sexuales. Amanda, la protagonista, se sitúa en un presente impreciso para rememorar una época de su vida marcada por el trauma personal, lo que la convierte en una ‘sujeto de evocación', el principal soporte memorialístico de la narración: "Esa noche ocurrió algo extraño, que entonces no llegué a entender bien. Unos años más tarde supe por qué algunos partían al extranjero y a otros, como a mí mismo padre y a Ramón, no los volvíamos a ver más" (Costamagna, 1996: 9). Sin embargo, a medida que avanza la trama, este efecto de evocación va disolviéndose con el desarrollo de la misma, en donde el tiempo pasado se vuelve presente e impregna al tiempo narrativo de enunciación. De este modo, se van haciendo frecuentes los diálogos en estilo directo. Por otro lado, el presente enunciativo no se define y no se vuelve sobre él, concentrándose la narración solamente en el pasado rememorado y así la memoria adquiere autonomía en relación al presente. Entendemos entonces cómo formalmente la ficción se apropia del ejercicio memorialístico, utilizando sólo parcialmente el registro del género referencial autobiográfico. La construcción del trauma de Amanda, a partir del discurso del silenciamiento, permite que emerja una 'testigo inmanente', o sea, una testigo de su propio trauma, incapaz de trascender-lo.

Respecto a Jeftanovic y Escenario de guerra (2000), observamos el trabajo de la posmemoria impedido por la no-elaboración del trauma paterno, por lo cual éste no puede compartir su testimonio, sino que muy por el contrario, la margina de cualquier posibilidad de acceso a éste. La pequeña Tamara crece conviviendo con los síntomas postraumáticos de 
su padre, como por ejemplo, acting-out, pesadillas, manías compulsivas, entre otros, los que va incorporando y corporizando en su propia vida. En la obra advertimos una ilusión anamnética sostenida, por un lado, en la configuración de un 'testigo-autor' que narrativiza la 'memoria traumática', y por otro, en el espacio biográfico desplegado. El testimonio de Tamara es tanto sobre su padre y su experiencia del trauma, como acerca de la apropiación traumática de éste y las consecuencias actuales de ello, siendo la principal, la indefinición identitaria de esta personaje. En segundo lugar, el espacio biográfico se encarga de insertar una accidentada linealidad en el relato de la vida de Tamara, marcada por diversos hitos traumáticos que son narrados sucesivamente. La fragmentación del relato memorialístico está dada por las repeticiones y acting-out derivada de su estado de trauma constante, lo cual no impide el acceso del lector a su relato de vida. Como en toda autobiografía, existe un hito que marca un antes y un después, que en este caso es el viaje definitivo al 'otro continente', aquel territorio geográfico que marca los orígenes del trauma de su padre, en donde encuentra al hermano gemelo de éste 'testigo contactual', quien le revela el acontecimiento límite que jamás le fue testimoniado por su propio padre: el secreto familiar. Desde esta instancia de revelación, el espacio biográfico se constituye a partir de distintas transgresiones al código de la biografía como 'escritura del yo', así por ejemplo, no se gesta una reducción de la distancia entre autor, narrador y personaje, ya que aquí la firma legitimadora del autor no importa. Si bien existen algunas conexiones entre Tamara y Andrea Jeftanovic, como el hecho de ser descendiente de un padre yugoslavo y una madre judía, no es lo que prevalece referencialmente, es decir, el relato no está sujeto a la realidad del autor, sino que más bien, a la verosimilitud de la personaje y la coherencia de su historia. Lo que respalda la autonomía del relato autobiográfico es el reflejo especular del acto de escribir sobre su vida dentro de la ficción narrativa, lo cual se simboliza en el 'cuaderno azul' de Tamara, en el cual narra el horror de sus experiencias, asociadas al placer de la escritura. "Fundé mi patria en un cuaderno azul, donde no soy minoría” (30). En este autónomo espacio, Tamara logra conectarse con su historia, a partir de una estética de lo íntimo que se levanta en la compleja relación entre horror y placer: "Mis palabras son un grito en la hoja. A medida que escribo sobre mi vida, dejo de formar parte de ella, creando otra existencia entre líneas" (161). 
En suma, en esta 'ficciones narrativas' podemos apreciar un trabajo de apropiación flexible de los géneros referenciales para construir una ilusión anamnética formal desde diversos gestos de validación y/o cuestionamiento de dichos registros escriturales, produciendo una reactualización del ejercicio de la memoria social en la escritura literaria ${ }^{10}$. Dicha reactualización considera las voces del trauma individual y colectivo, configurando distintos niveles de 'testigos', los que vienen a complejizar el sistema de rememoración de la novela.

\section{Referencias}

Agamben, G. (2000). Lo que queda de Auschwitz. El archivo y el testigo. Valencia: Editorial Pretextos.

Andruetto, M. T. (2009). La mujer en cuestión. Buenos Aires: Editorial De Bolsillo.

Avelar, I. (2000). Alegorías de la derrota: La ficción postdictatorial y el trabajo del duelo. Santiago, Chile: Cuarto Propio.

Cánovas, R.; Sherman Filer, J. (2008). Voces femeninas en Chile: miradas sobre el ser mosaico. Estudios Filológicos, 43, 19-37.

Carreño, R. (2009). Memorias del nuevo siglo: jóvenes, trabajadores y artistas en la novela chilena reciente. Santiago, Chile: Cuarto Propio.

Costamagna, A. (1996). En voz baja. Santiago, Chile: Lom.

Dalmaroni, M. (2004). La palabra justa. Literatura, crítica y memoria en la Argentina (1960-2002). Recuperado de http://www.juangelman.net/wpcontent/uploads/2011/11/Dalmaroni-La-palabra-justa.pdf

Di Marco, J. (2003). Ficción y memoria en la narrativa argentina actual: La escritura como táctica. V Congreso Internacional Orbis Tertius de Teoría y Crítica Literaria, La Plata. Recuperado de http://www.fuentesmemoria. fahce.unlp.edu.ar/trab_eventos/ev.11/ev.11.pdf

Felman, S. y Laub, D. (1992). Bearing witness or the vicissitudes of listening. En Testimony: Crises of witnessing in literature, psychoanalysis, and history (pp. 201-234). New York: Routledge.

${ }^{10}$ Consideramos que para un estudio amplio de la posición que ocupan estas 'ficciones narrativas de la memoria' frente a la tradición de la novela y su relación con la escritura del trauma, sería pertinente observar las influencias de escritores 'sujetos de la experiencia' propiamente tal, como es el caso de Primo Levi o Judith Klein, para apreciar los modos en que estos nuevos escritores, sujetos de la 'posmemoria', asumen el trauma. 
Gramuglio, M. T. (2002). Políticas del decir y formas de la ficción. Punto de Vista, 74, 9-14.

Heker, L. (2004). El fin de la historia. Buenos Aires: Punto de Lectura.

Jeftanovic, A. (2000). Escenario de guerra. Santiago, Chile: Alfaguara.

La Capra, D. (2005). Escribir la historia, escribir el trauma. Buenos Aires: Nueva Visión.

La Capra, D. (2006). Historia en tránsito. Experiencia, identidad, teoría crítica. Buenos Aires: Fondo de Cultura Económica.

Lazzara, M. J. (2007). Prismas de la memoria: narración y trauma en la transición chilena. Santiago, Chile: Cuarto Propio.

Martínez Bonati, F. (2001). La ficción narrativa. Su lógica y ontología. Santiago, Chile: Lom.

Mercado, T. (2003). Narrar después. Rosario: Beatriz Viterbo.

Mignolo, W. (1986). Teoría del texto e interpretación de textos. México: UNAM.

Miraux, J.-Ph. (2005). La autobiografía: las escrituras del yo. Buenos Aires: Nueva Visión.

Ochando Aymerich, C. (1998). La memoria en el espejo: Aproximaciones a la escritura testimonial. Barcelona: Anthropos.

Peris Blanes, J. (2009). La imposible voz. Memoria y representación de los campos de concentración en Chile. La posición del testigo. Santiago, Chile: Cuarto Propio.

Pratt, M. L. (2000). No interrumpas: Las mujeres y el ensayo latinoamericano. Debate Feminista. Recuperado de http://www.ignaciodarnaude.com/textos _diversos/Ocampo,M.L.Pratt.pdf

Richard, N. (1998). Residuos y metáforas. (Ensayos de crítica cultural sobre el Chile de la Transición). Santiago, Chile: Cuarto Propio.

Stern, S. (2000). De la memoria suelta a la memoria emblemática. Hacia el recordar y el olvidar como proceso histórico (Chile 1973-1998). En Garcés, M.; Milos, P.; Olguín, M.; Pinto, J.; Rojas, M. T. y Urrutia, M. (comps.), Memoria para un nuevo siglo: Chile, miradas a la segunda mitad del siglo $X X$ (pp. 11-33). Santiago, Chile: Lom.

Strejilevich, N. (2006). El arte de no olvidar: Literatura testimonial en Chile, Argentina y Uruguay entre los 80 y los 9o. Buenos Aires: Catálogos.

Volek, E. (2000). Testimonio, y otras ficciones: A propósito de un género que quería ser profético. Literatura: teoría, historia, crítica, 2, 47-6o. 